\title{
THE FASANELLA-SERVAT PROCEDURE: A RETROSPECTIVE STUDY
}

\author{
R. SAMPATH, D. C. SAUNDERS and B. LEATHERBARROW \\ Manchester
}

\begin{abstract}
SUMMARY
A retrospective study was carried out to assess the success of the Fasanella-Servat procedure for the management of the aponeurotic defect type of adult ptosis. Eighteen procedures were carried out on 16 patients. The mean follow-up was 3 years. The success rate of the operation was $28 \%$ with a mean residual tarsal plate height of $5 \mathrm{~mm}$ with a very variable tarsal plate contour.
\end{abstract}

The Fasanella-Servat procedure ${ }^{1}$ was originally described in 1961 as a form of surgical correction for minimal ptosis. It was designed to shorten the operating time and simplify the technical difficulties for a general ophthalmic surgeon who performs ptosis surgery 'occasionally'.

The aim of this study was to assess the efficacy, long-term results and complications of the Fasanella-Servat procedure for the management of adult patients with aponeurotic defect ptosis.

\section{MATERIALS AND METHODS}

The operation records from the 5 year period 1987-1992 were examined to determine the number of adult patients who had undergone a Fasanella-Servat procedure for an aponeurotic defect at our institution. Of the 22 patients who had undergone this procedure, 16 were able to return to the oculoplastic clinic to undergo a clinical assessment. The remaining 6 patients were not contactable. The case records of these patients were reviewed. The age range of the 16 patients was 54-85 years (mean 71 years). There were 11 women and 5 men. Two of the patients had undergone a bilateral procedure. The cause of ptosis was involutional in 12 patients. The remaining patients had developed signs of a levator aponeurosis disinsertion following cataract surgery. The degree of pre-operative ptosis varied

From: Manchester Royal Eye Hospital, Oxford Road, Manchester M13 9WH, UK.

Correspondence to: R. Sampath, 5 Devonshire House, Devonshire Avenue, Sutton, Surrey SM2 5JJ, UK. from $2 \mathrm{~mm}$ to $5 \mathrm{~mm}$; the levator function varied from $10 \mathrm{~mm}$ to $15 \mathrm{~mm}$. The height of the tarsal plate had not been assessed pre-operatively.

The 16 patients who attended for review underwent a complete ophthalmic examination with particular attention being given to measurement of the palpebral apertures and the residual tarsal plate height. The follow-up period for these patients varied from 9 months to 6 years (mean 3 years). The operation was considered to be successful if the lid was within $1 \mathrm{~mm}$ of the desired height.

\section{RESULTS}

Of the 18 procedures performed, it was noted in the case records that $9(50 \%)$ of them were associated with a symptomatic superior corneal abrasion in the immediate post-operative period. This had necessitated more frequent and prolonged follow-up in these cases. The abrasions healed with no serious sequelae. On the basis of our clinical assessment and from an examination of the case notes, $13(72 \%)$ eyelids had an undercorrection and/or recurrence of the ptosis while only $5(28 \%)$ had a successful result. The residual tarsal plate height varied from only 3 $\mathrm{mm}$ to $10 \mathrm{~mm}$ (mean $5 \mathrm{~mm}$ ). The contour of the superior aspect of the tarsal plate was very variable (Fig. 1). There were no other complications from the procedure. Of the 13 lids with an undercorrection or recurrence of ptosis, 8 have since undergone an anterior approach levator aponeurosis advancement with a successful result and 5 are awaiting a similar procedure.

\section{DISCUSSION}

Involutional or post-cataract ptosis has been shown to be due to aponeurosis disinsertion or dehiscence. $^{2-4}$ It has been suggested that the levator aponeurosis plays no role in the success of the Fasanella-Servat procedure. ${ }^{5}$ Other mechanisms such as tarsal shortening, cicatricial contraction of the wound, plication or advancement on the tarsus of 

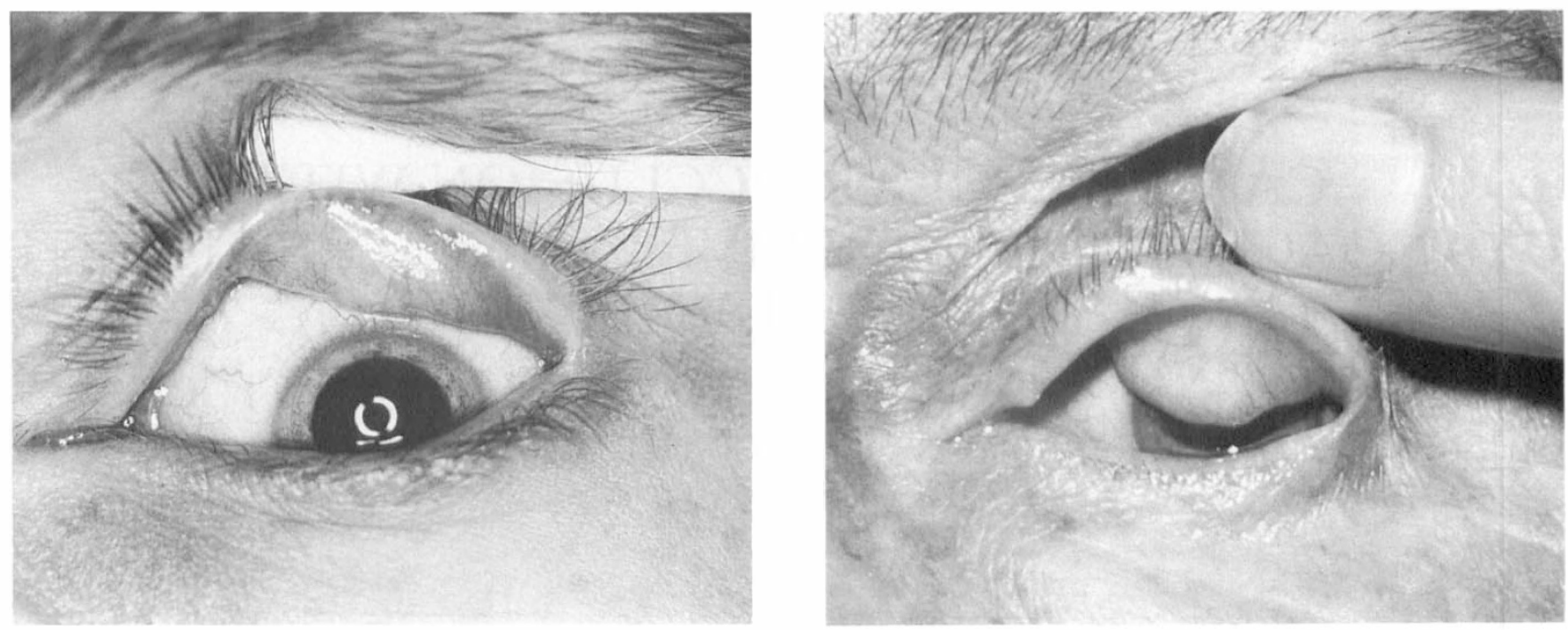

Fig. 1. The variable residual tarsal plate height and contour in two of our study patients.

the Muller's muscle-levator aponeurosis complex have been postulated for the success of the procedure. The way the procedure corrects minimal ptosis is probably a combination of these factors. ${ }^{6}$

The pre-operative assessment, management decision and surgery in our study were undertaken by general ophthalmologists. The success rate and the immediate post-operative complication of corneal abrasion in our study compare very poorly with other studies. $^{5-7}$ Poor pre-operative assessment and inappropriate case selection may be responsible for this. The indication for the Fasanella-Servat procedure should be a mild ptosis ( $2 \mathrm{~mm}$ or less) with good levator function $(10 \mathrm{~mm}$ or more $){ }^{8}$ The general ophthalmologist performing very occasional ptosis surgery may be tempted to select this procedure inappropriately for significant degrees of ptosis because of its speed and simplicity.

Given that the Fasanella-Servat procedure does not deal with the aponeurosis as such, it is not surprising that it does not have a high rate of success in significant ptosis of this nature. Though a number of modifications of the procedure have been described, the majority involve resection of the tarsal plate. ${ }^{5,7,9}$ The procedure described by Putterman et al. ${ }^{9}$ involves resection of the conjunctiva and Muller's muscle avoiding the tarsal plate. This to some extent removes the technical simplicity of the Fasanella-Servat procedure but again does not deal with the primary problem of aponeurosis dehiscence. Because the Fasanella-Servat procedure does not deal with the primary problem, and because of the possible later complications of resecting the tarsal plate, this procedure may not be appropriate for dealing with aponeurosis disinsertion or dehiscence type of ptosis.

Reoperation, involving anterior approach levator aponeurosis advancement, in these cases was problematic due to scarring and loss of a very variable portion of the tarsal plate.

We conclude that the Fasanella-Servat procedure, although popular among general ophthalmologists, has limited efficacy in the management of patients with significant ptosis (greater than $2 \mathrm{~mm}$ ), particularly those in whom the primary problem is with the levator aponeurosis. The procedure tends to be selected inappropriately for degrees of ptosis that exceed 1-2 mm. If, as discussed previously, strict indication criteria are observed this procedure can produce satisfactory results.

Key words: Fasanella-Servat procedure, Ptosis, Surgery.

\section{REFERENCES}

1. Fasanella RM, Servat J. Levator resection for minimal ptosis: another simplified operation. Arch Ophthalmol 1961;65:493-6.

2. Anderson RL, Beard C. The levator aponeurosis: attachments and their clinical significance. Arch Ophthalmol 1977;95:1437-41.

3. Collin JRO, Beard C, Wood I. Experimental and clinical data on the insertion of the levator palpebrae superioris muscle. Am J Ophthalmol 1978;85:792-801.

4. Paris GL, Quickert MH. Disinsertion of the aponeurosis of the levator palpebrae superioris muscle after cataract extraction. Am J Ophthalmol 1976;81:337-40.

5. Beard C. Blepharoptosis repair by modified Fasanella-Servat operation. Am J Ophthalmol 1970; 69:850-7.

6. Buckman G, Jakobiec FA, Hyde K, et al. Success of the Fasanella-Servat operation independent of Muller's smooth muscle excision. Ophthalmology 1989;96:413-8.

7. Gupta VP, Aggarwal R, Mathur SP. Blepharoptosis repair by modified Fasanella-Servat operation: a large series of 50 cases. Ind J Ophthalmol 1992;40(30):86-9.

8. Collin JRO. A manual of systematic eyelid surgery. 2nd ed. Edinburgh: Churchill Livingstone, 1989:48.

9. Putterman AM, Urist MJ. Muller's muscle-conjunctival resection: technique for treatment of blepharoptosis. Arch Ophthalmol 1975;93:619-23. 